ANNALES

POLONICI MATHEMATICI

$82.1(2003)$

\title{
Two remarks on \\ non-zero constant Jacobian polynomial maps of $\mathbb{C}^{2}$
}

\author{
by NGuYen Van Chau (Hanoi)
}

\begin{abstract}
We present some estimates on the geometry of the exceptional value sets of non-zero constant Jacobian polynomial maps of $\mathbb{C}^{2}$ and their components.
\end{abstract}

1. Introduction. Recall that the exceptional value set $E_{h}$ of a polynomial mapping $h: \mathbb{C}^{m} \rightarrow \mathbb{C}^{n}$ is the smallest subset $E_{h} \subset \mathbb{C}^{n}$ such that the restriction $h: h^{-1}\left(\mathbb{C}^{n} \backslash E_{h}\right) \rightarrow \mathbb{C}^{n} \backslash E_{h}$ gives a locally trivial smooth fibration. The mysterious Jacobian conjecture (see $[\mathrm{BCW}]$ and $[\mathrm{E}]$ ), posed by Keller in 1939 and still open even in the two-dimensional case, asserts that a polynomial map of $\mathbb{C}^{n}$ with non-zero constant Jacobian must be a polynomial bijection. Consider a polynomial map $f=(P, Q): \mathbb{C}_{(x, y)}^{2} \rightarrow \mathbb{C}_{(u, v)}^{2}$ and define $J(P, Q):=P_{x} Q_{y}-P_{y} Q_{x}$. It is well known that if $J(P, Q) \equiv$ const $\neq 0$ but $f$ is not bijective, then the sets $E_{P}$ and $E_{Q}$ must be non-empty finite sets and $E_{f}$ is a curve so that each of its irreducible components is a polynomially parameterized curve (the image of a polynomial map from $\mathbb{C}$ into $\mathbb{C}^{2}$ ) (see, for example, [J1, J2]).

In this note we prove the following.

THEOREM 1. Suppose $f=(P, Q)$ is a polynomial map with non-zero constant Jacobian. Then $u_{0} \in E_{P}$ if and only if the line $u=u_{0}$ is tangent to an irreducible local branch of $E_{f}$.

Here, by saying that a line $L$ is tangent to an irreducible local branch curve $\gamma$ we mean that the intersection multiplicity of $L$ and $\gamma$ at their common point is larger than 1 , or equivalently, either the common point is a singular point of $\gamma$ or $L$ is the line tangent to $\gamma$ at this point.

2000 Mathematics Subject Classification: 14H07, 14R15.

Key words and phrases: exceptional value set, Jacobian conjecture.

Supported in part by the National Basic Program for Natural Sciences, Vietnam. 
This theorem leads to the following fact that may be used to consider the non-existence of non-zero constant Jacobian polynomial maps with exceptional value curve of a given type.

TheOREM 2. The exceptional value set of a polynomial map of $\mathbb{C}^{2}$ with non-zero constant Jacobian cannot be isomorphic to a curve composed of the images of polynomial maps of the form $t \mapsto\left(t^{k}, q(t)\right), k \in \mathbb{N}, q(t) \in \mathbb{C}[t]$.

A special property of a curve with irreducible components given by parameterizations in Theorem 2 is that its irreducible local branches may be tangent to the line $u=0$ only. Simply connected curves are simple examples of such curves. This can be easily deduced from Lin-Zaĭdenberg's theorem on simply connected curves (Theorem B in [ZL]).

Corollary 3. The exceptional value set of a polynomial map of $\mathbb{C}^{2}$ with non-zero constant Jacobian cannot be a simply connected curve.

Proofs of Theorems 1 and 2 will be given in Sections 3 and 4 .

2. Preliminaries. In this section, we present some elementary facts which will be used in the proof of Theorem 1 .

(i) We will work with finite fractional power series with parameter $\xi$ of the form

$$
\varphi(x, \xi)=\sum_{k=1}^{K-1} a_{k} x^{n_{k} / m}+\xi x^{n_{K} / m}, \quad 0 \neq a_{k} \in \mathbb{C}, n_{k} \in \mathbb{Z}, m \in \mathbb{N},
$$

with $n_{1}>\ldots>n_{K}$ and $\operatorname{gcd}\left(\left\{n_{k}: k \leq K\right\}\right)=1$.

Let $h(x, y)$ be a non-constant primitive polynomial monic in $y$ with $\operatorname{deg}_{y} h=\operatorname{deg} h$. A series $\varphi(x, \xi)$ in (1) is called a Newton-Puiseux type of $h$ if

$$
h(x, \varphi(x, \xi))=h_{\varphi}(\xi)+\text { lower terms in } x, \quad h_{\varphi} \in \mathbb{C}[\xi], \operatorname{deg} h_{\varphi}>0 .
$$

Define $\operatorname{mult}(\varphi):=m$ and $\operatorname{ord}(\varphi):=n_{K}$.

The Newton-Puiseux types of $h(x, y)$ can be constructed from the Newton-Puiseux expansions at infinity of the curve $h(x, y)=0$. In fact, if $y=$ $y(x)$ is such an expansion, then there is a unique Newton-Puiseux type $\varphi$ of $h$ and a number $\xi_{0} \in \mathbb{C}$ such that $y(x)=\varphi\left(x, \xi_{0}\right)+$ lower terms in $x$.

For a Newton-Puiseux type $\varphi$ of $h$ the rational map $\Phi: \mathbb{C} \times \mathbb{C} \rightarrow \mathbb{C P}^{2}$ defined by

$$
\Phi(t, \xi):=\left(t^{-\operatorname{mult}(\varphi)}, \varphi\left(t^{-\operatorname{mult}(\varphi)}, \xi\right)\right)
$$

determines an unbranched $i_{\varphi}$-sheeted covering from $\mathbb{C}^{*} \times \mathbb{C}$ onto $\Phi\left(\mathbb{C}^{*} \times \mathbb{C}\right)$ $\subset \mathbb{C}^{2}$, where $i_{\varphi}:=\operatorname{mult}(\varphi) / \operatorname{gcd}\left\{n_{k}: k=1, \ldots, K-1\right\}$. Hence, one can use the polynomial $H_{\varphi}(t, \xi):=h \circ \Phi(t, \xi)$ as a kind of "extension" of $h(x, y)$. 
Lemma 4 (see [C, Theorem 2.4]). Suppose $h(x, y)$ is a primitive polynomial monic in $y$. Then $c \in E_{h}$ if and only if $c$ is either a critical value of $h$ or a critical value of $h_{\varphi}(\xi)$ for any Newton-Puiseux type $\varphi$ of $h$.

In fact, by Newton's theorem the polynomial $h(x, y)-c$ can be factorized as

$$
h(x, y)-c=C \prod_{u}(y-u(x)),
$$

where the product runs over all Newton-Puiseux expansions at infinity of the curve $h=c$. Substituting $y=\varphi(x, \xi)$ into this representation, one can see that a number $\alpha \in \mathbb{C}$ is a zero of $h_{\varphi}(\xi)-c=0$ with multiplicity $n$ if and only if the level $h=c$ has exactly $n$ Newton-Puiseux expansions at infinity of the form $\varphi(x, \alpha)+$ lower terms in $x$. Thus, Puiseux data at infinity of the curves $h=c$ must be changed when $c$ is a critical value of $h_{\varphi}$.

(ii) Consider a polynomial map $f=(P, Q): \mathbb{C}^{2} \rightarrow \mathbb{C}^{2}$. By definition the exceptional value set $E_{f}$ is composed of the critical value set of $f$ and the so-called non-proper value set $A_{f}$ of $f$, the set of all values $a \in \mathbb{C}^{2}$ such that there exists a sequence $\mathbb{C}^{2} \ni p_{i} \rightarrow \infty$ with $f\left(p_{i}\right) \rightarrow a$. By [J1], the non-proper value set $A_{f}$, if non-empty, is a curve composed of the images of some polynomial maps from $\mathbb{C}$ into $\mathbb{C}^{2}$.

A series $\varphi(x, \xi)$ in (1) is called a dicritical series of $f$ if

$$
f(x, \varphi(x, \xi))=f_{\varphi}(\xi)+\text { lower terms in } x, \quad \operatorname{deg} f_{\varphi}>0 .
$$

LEMMA 5.

$$
A_{f}=\bigcup_{\varphi \text { is a dicritical series of } f} f_{\varphi}(\mathbb{C}) .
$$

Proof. Let $\varphi$ be a dicritical series of $f$ and $\Phi(t, \xi)$ be as in (2). The map $\Phi$ sends $\mathbb{C}^{*} \times \mathbb{C}$ to $\mathbb{C}^{2}$ and the line $\{0\} \times \mathbb{C}$ to the line at infinity of $\mathbb{C P}^{2}$. Then the polynomial map $F_{\varphi}(t, \xi):=f \circ \Phi(t, \xi)$ maps the line $\{0\} \times \mathbb{C}$ into $A_{f} \subset \mathbb{C}^{2}$. Therefore, $f_{\varphi}(\mathbb{C})$ is an irreducible component of $A_{f}$, since $\operatorname{deg} f_{\varphi}>0$.

Conversely, assume that $\ell$ is an irreducible component of $A_{f}$. By definition we can choose a smooth point $\left(u_{0}, v_{0}\right)$ of $\ell$ and an irreducible branch at infinity $\gamma$ of the curve $P=u_{0}$ (or $Q=v_{0}$ ) whose image $f(\gamma)$ is a branch curve intersecting $\ell$ transversally at $\left(u_{0}, v_{0}\right)$. Let $\varphi(x, \xi)$ be the NewtonPuiseux type of $P$ corresponding to a Newton-Puiseux expansion at infinity of $\gamma$. Then by definitions we can verify that $\varphi$ is a dicritical series of $f$ and $f_{\varphi}(\mathbb{C})=\ell$.

3. Proof of Theorem 1. We consider a polynomial map $f=(P, Q)$ : $\mathbb{C}^{2} \rightarrow \mathbb{C}^{2}$ with non-zero constant Jacobian. Fix affine coordinates $(x, y)$ so 
that $P$ and $Q$ are monic in $y$. For a series $\varphi$ in (1) write

$$
\begin{aligned}
& P(x, \varphi(x, \xi))=p_{\varphi}(\xi) x^{a_{\varphi} / \operatorname{mult}(\varphi)}+\text { lower terms in } x, \\
& Q(x, \varphi(x, \xi))=q_{\varphi}(\xi) x^{b_{\varphi} / \operatorname{mult}(\varphi)}+\text { lower terms in } x,
\end{aligned}
$$

where $p_{\varphi} \neq 0$ and $q_{\varphi} \neq 0$.

Lemma 6. (i) Let $\varphi$ be a Newton-Puiseux type of $P$. If $\varphi$ is not a dicritical series of $f$, then $\operatorname{deg} p_{\varphi}(\xi)=1, q_{\varphi}(\xi) \equiv$ const $\neq 0$ and $b_{\varphi}>0$.

(ii) A dicritical series of $f$ must be a Newton-Puiseux type of both $P$ and $Q$.

Proof. (i) First, we will show that

$$
\operatorname{deg} p_{\varphi}=1, \quad q_{\varphi} \equiv \text { const } \neq 0 .
$$

Differentiating $F\left(t^{-\operatorname{mult}(\varphi)}, \varphi\left(t^{-\operatorname{mult}(\varphi)}, \xi\right)\right)$, since $a_{\varphi}=0$ and $b_{\varphi} \neq 0$ we have

$$
\operatorname{mult}(\varphi) J(P, Q) t^{\operatorname{ord}(\varphi)-\operatorname{mult}(\varphi)-1}=-b_{\varphi} \dot{p}_{\varphi} q_{\varphi} t^{-b_{\varphi}-1}+\text { higher terms in } t .
$$

Since $J(P, Q) \equiv$ const $\neq 0$ and $\operatorname{deg} p_{\varphi}>0$, we get $(*)$.

Now, assume to the contrary that $b_{\varphi}<0$. Then there exists a NewtonPuiseux root at infinity $u(x)$ of the curve $Q=0$ such that $u(x)=\varphi\left(x, \xi_{0}\right)+$ lower terms in $x$. Let $\psi(x, \xi)$ be the Newton-Puiseux type of $Q$ corresponding to $u(x)$. Obviously, $a_{\psi}>a_{\varphi}=0$ and hence $\psi$ is not a dicritical series of $f$. Furthermore, $\varphi(x, \xi)=\psi(x, \alpha)+$ lower terms in $x$ for a zero $\alpha$ of $p_{\psi}(\xi)$. This is impossible, since $p_{\psi}(\xi) \equiv$ const $\neq 0$ by applying $(*)$ to the Newton-Puiseux type $\psi$ of $Q$. Thus, we get $b_{\varphi}>0$.

(ii) This is obtained from (i) and the definitions.

Proof of Theorem 1. If $E_{f}=\emptyset$, then $f$ is bijective and $E_{P}=E_{Q}=\emptyset$. Hence, we need only consider the situation when $E_{f} \neq \emptyset$. In this situation $E_{f}=A_{f}$, since $f$ has no singularity.

First, suppose the line $u=u_{0}$ is tangent to an irreducible local branch curve of an irreducible component $\ell$ of $A_{f}$. By Lemma 5 , there is a dicritical series $\varphi$ of $f$ such that $\ell$ is the image of $f_{\varphi}:=\left(p_{\varphi}, q_{\varphi}\right)$. By Lemma 6(ii) the series $\varphi$ is a Newton-Puiseux type of both $P$ and $Q$. Since the line $u=u_{0}$ is tangent to $\ell, u_{0}$ must be a critical value of $p_{\varphi}$. Hence, by Lemma 4 the number $u_{0}$ is an exceptional value of $P$.

Conversely, let $u_{0} \in \mathbb{C}$ and $L:=\left\{u=u_{0}\right\}$. Assume that $L$ intersects transversally each of the irreducible local branches of $A_{f}$ located at points in $L \cap A_{f}$. We have to show that $u_{0}$ cannot be an exceptional value of $P$. In view of Lemmas 4 and 6 we only need to verify that $u_{0}$ is a regular value of $p_{\varphi}(\xi)$ for every dicritical series $\varphi$ of $f$.

Let $\varphi$ be such a series and $\ell:=f_{\varphi}(\mathbb{C})$, which is an irreducible component of $A_{f}$ by Lemma 5. Let $\left(u_{0}, v_{0}\right) \in \ell$ and $\xi_{0} \in \mathbb{C}$ with $f_{\varphi}\left(\xi_{0}\right)=\left(u_{0}, v_{0}\right)$. We 
have to show that $\dot{p}_{\varphi}\left(\xi_{0}\right) \neq 0$.

Consider the polynomial map $F: \mathbb{C} \times \mathbb{C} \rightarrow \mathbb{C}^{2}$ given by $F(t, \xi):=f \circ\left(t^{-\operatorname{mult}(\varphi)}, \varphi\left(t^{-\operatorname{mult}(\varphi)}, \xi\right)\right)=\left(p_{\varphi}, q_{\varphi}\right)(\xi)+$ higher terms in $t$. For this map $F(\{0\} \times \mathbb{C})=\ell$ and

$$
\operatorname{det} D F(t, \xi)=-\operatorname{mult}(\varphi) J(P, Q) t^{\operatorname{ord}(\varphi)-\operatorname{mult}(\varphi)-1} .
$$

Since $J(P, Q) \equiv$ const $\neq 0, F$ has a singularity on the line $t=0$ only.

Let $\gamma:=f_{\varphi}\left(\left\{\xi:\left|\xi-\xi_{0}\right|<\varepsilon\right\}\right.$ for some $\varepsilon>0$ small enough. By assumption the line $L$ intersects $\gamma$ transversally at $\left(u_{0}, v_{0}\right)$. So, we can choose a sufficiently small neighborhood $U$ of $\left(u_{0}, v_{0}\right)$ so that $\gamma:=\ell \cap U$ is a smooth branch curve parameterized by $v=v_{0}+h\left(u-u_{0}\right)$ for a homeomorphic function $h, h(0)=0$. Define new coordinates $(\bar{u}, \bar{v})=\left(u-u_{0}, v-v_{0}-h\left(u-u_{0}\right)\right)$ in $U$ and $(\bar{t}, \bar{\xi})=\left(t, \xi-\xi_{0}\right)$ in a sufficiently small neighborhood $V$ of $\left(0, \xi_{0}\right)$. Let $\bar{F}=\left(\bar{F}_{1}, \bar{F}_{2}\right)$ be the representation of $F$ in these coordinates. Then

$$
\bar{F}_{1}(\bar{t}, \bar{\xi})=p_{\varphi}(\bar{\xi})-u_{0}+\text { higher terms in } \bar{t}
$$

$\bar{F}(0,0)=(0,0), \bar{F}(\{\bar{t}=0\}) \subset \gamma=\{\bar{v}=0\}$ and $\operatorname{det} D \bar{F}(\bar{t}, \bar{\xi}) \neq 0$ for $\bar{t} \neq 0$. Then, by examining the Newton diagrams of $\bar{F}_{1}, \bar{F}_{2}$ and $\operatorname{det} D \bar{F}$ we can verify that

$$
\bar{F}(\bar{t}, \bar{\xi})=\left(\bar{\xi} u_{1}(\bar{t}, \bar{\xi})+\bar{t} u_{2}(\bar{t}, \bar{\xi}), \bar{t}^{k} v_{1}(\bar{t}, \bar{\xi})\right),
$$

where $u_{1}, u_{2}$ and $v_{1}$ are homeomorphic functions defined in $V, u_{1}(0,0) \neq 0$ and $v_{1}(0,0) \neq 0$ (see, for example, [O, Lemma 4.1]). From (4) and (5) it follows that $\dot{p}_{\varphi}\left(\xi_{0}\right) \neq 0$.

Remark. From Lemmas 6(ii) and 5 one can easily see that the exceptional value set $E_{f}$ of a non-singular polynomial map $f$ cannot contain an irreducible component isomorphic to a line.

4. Proof of Theorem 2. Let $f=(P, Q)$ be the representation of $f$ in a coordinate system in which $E_{f}$ consists of the images of some polynomial maps of the form $t \mapsto\left(t^{k}, q(t)\right), k \in \mathbb{N}$. By applying Theorem 1 we find that $E_{P} \subset\{0\}$. As $f$ has no singularity, $P$ is a non-singular primitive polynomial. Then from Suzuki's equality

$$
\sum_{c \in \mathbb{C}}\left(\chi_{c}-\chi\right)=1-\chi
$$

(see $[\mathrm{S}]$ ) we get $\chi_{0}=1$. Here, $\chi_{c}$ and $\chi$ indicate the Euler-Poincaré characteristics of the fiber $P=c$ and of the generic fiber of $P$, respectively. Since the curve $P=0$ is smooth, it has one connected component $\ell$ diffeomorphic to $\mathbb{C}$. This component $\ell$ must be isomorphic to $\mathbb{C}$ by the Abhyankar-Moh Theorem $[\mathrm{AM}]$ and the restriction of $f$ to $\ell$ must be injective. Then, as ob- 
served by Gwoździewicz [G], $f$ must be bijective. This is impossible, since $E_{f} \neq \emptyset$.

Acknowledgments. The author wishes to thank Prof. V. H. Ha and the referee for many valuable suggestions and useful discussions.

\section{References}

[AM] S. S. Abhyankar and T. T. Moh, Embeddings of the line in the plane, J. Reine Angew. Math. 276 (1975), 148-166.

[BCW] H. Bass, E. Connell and D. Wright, The Jacobian conjecture: reduction of degree and formal expansion of the inverse, Bull. Amer. Math. Soc. (N.S.) 7 (1982), 287-330.

[C] Nguyen Van Chau, Non-zero constant Jacobian polynomial maps of $\mathbb{C}^{2}$, Ann. Polon. Math. 71 (1999), 287-310.

[E] A. van den Essen, Polynomial Automorphisms and the Jacobian Conjecture, Progr. Math. 190, Birkhäuser, Basel, 2000.

[G] J. Gwoździewicz, Injectivity on one line, Bull. Soc. Sci. Łódź Sér. Rech. Déform. 15 (1993), 59-60.

[J1] Z. Jelonek, The set of points at which a polynomial map is not proper, Ann. Polon. Math. 58 (1993), 259-266.

[J2] - Testing sets for properness of polynomial mappings, Math. Ann. 315 (1999), $1-35$.

[O] S. Yu. Orevkov, On three-sheeted polynomial mappings of $\mathbb{C}^{2}$, Izv. Akad. Nauk SSSR 50 (1986), 1231-1240 (in Russian).

[S] M. Suzuki, Propriétés topologiques des polynômes de deux variables complexes et automorphismes algébriques de l'espace $\mathbb{C}^{2}$, J. Math. Soc. Japan 26 (1974), 241-257.

[ZL] M. G. Zaĭdenberg and V. Ya. Lin, An irreducible simply connected algebraic curve in $\mathbb{C}^{2}$ is equivalent to a quasihomogeneous curve, Soviet Math. Dokl. 28 (1983), 200-204.

Hanoi Institute of Mathematics

P.O. Box 631, Boho 10000, Hanoi, Vietnam

E-mail: nvchau@thevinh.ncst.ac.vn

Reçu par la Rédaction le 24.4.2002

Révisé le 22.1.2003 